\title{
Zum Mechanismus der Reaktion von 1,6-Methano-[10]annulen mit Elektrophilen")
}

\author{
Franz Effenberger* und Herbert Klenk ${ }^{21}$
}

Institut für Organische Chemie, Biochemie und Isotopenforschung der Universität Stuttgart, D-7000 Stuttgart 80, Pfaffenwaldring 55

Eingegangen am 4. Juli 1975

Mit Chlorsulfonylisocyanat reagiert 1,6-Methano-[10]annulen (1) ausschließlich unter Bildung des Substitutionsproduktes 7; ein Cycloadditionsprodukt ist nicht nachzuweisen. Beweisend für eine zweistufige Additionsreaktion von Brom an 1 über kationische Zwischenverbindungen ist die Bildung des Dibrom-dichlor-Adduktes 10 in Gegenwart von Triäthylbenzylammoniumchlorid als Konkurrenznucleophil. Bei der Nitrierung von 1 mit Distickstoffpentoxid bei $-110^{\circ} \mathrm{C}$ entstehen NMR-spektroskopisch nachweisbare Additionsprodukte. Mit Collidin/Nitroniumtrifluormethansulfonat wird 1 nahezu ausschließlich in 2-Stellung nitriert, während mit anderen Nitrierungsagentien Gemische aus den Isomeren 5 und 6 entstehen.

\section{On the Mechanism of the Reaction of 1,6-Methano-[10|annulene with Electrophiles ")}

Chlorosulfonyl isocyanate reacts with 1,6-methano-[10]annulene (1) to give only the substitution product 7 ; cycloaddition products are not observed. The formation of the dibromo dichloro adduct 10 by bromination of 1 with $\mathrm{Br}_{2}$ in the presence of triethylbenzylammonium chloride as a competitive nucleophil provides evidence for a two-step addition via a cationic intermediate. Upon nitration of 1 with dinitrogen pentoxide at $-110^{\circ} \mathrm{C}$ addition products are formed which can be characterized by n.m.r. spectroscopy. With collidine/nitronium trifluoromethanesulfonate, 1 is nitrated almost exclusively in the 2-position to form 5 . With other nitration agents, however, mixtures of 5 and 6 are formed.

Alle grundlegenden Arbeiten zum 1,6-Methano-[10]annulen (1) wurden von Vogel und Mitarbb. ${ }^{3)}$ durchgeführt $^{3 a)}$. Im Vordergrund der Untersuchungen standen dabei Synthese und Studium des Grundzustandes ${ }^{4 a-z)}$.

"13. Mitteil. zur elektrophilen Aromatensubstitution; 12. Mitteil.: K. Huthmacher, G. König und $F$. Effenberger, Chem. Ber. 108, 2947 (1975).

2) Teil der Dissertation H. Klenk, Univ. Stuttgart 1974.

3) E. Vogel, Chimia 22, 21 (1968).

3a) Unsere Untersuchungen wurden mit Herrn Professor E. Vogel abgestimmt, dem wir an dieser Stelle für sein Entgegenkommen, seine Anregungen und seine Hilfe herzlich danken.

4) 4a) F. C. Gerson, E. Heilbronner, W. A. Böll und E. Vogel, Helv. Chim. Acta 48, 1494 (1965) (ESR-Spektren). - ${ }^{4 b)}$ H.Günther, Z. Naturforsch., Teil B 20, 948 (1965) ('H-NMR-Spektren). ${ }_{4 c)}$ M. Dobler und J. D. Dunitz, Helv. Chim. Acta 48, 1429 (1965) (Röntgenstrukturanalyse). 4d) R. Boschi, W. Schmidt und J.-C. Gfeller, Tetrahedron Lett. 1972, 4107 (Photoelektronenspektrum). - 4e) H. R. Blattmann, W. A. Böll, E. Heilbronner, G. Hohlneicher, E. Vogel und I.-P. Weber, Helv. Chim. Acta 49, 2017 (1966) (Elektronenspektren). $-{ }^{41)}$ W. Bremser, R. Hagen, E. Heilbronner und E. Vogel, ebenda 52, 418 (1969) (Bildungsenthalpie). - ${ }^{48}$ ) W. Bremser, H. T. Grunder, E. Heilbronner und E. Vogel, ebenda 50, 84 (1967) (Dipolmomente). 
Bei den im Kölner Arbeitskreis durchgeführten Reaktionen mit 1 waren jedoch noch einige Probleme offen, deren Klärung im Zusammenhang mit unseren Untersuchungen zur elektrophilen Aromatensubstitution wegen der Mittelstellung der Annulene zwischen Polyolefinen und Aromaten interessierte ${ }^{4 a-k}$.

1 besitzt eine große thermische Stabilität und reagiert erst bei $130^{\circ} \mathrm{C}$ mit Maleinsäureanhydrid unter Cycloaddition ${ }^{31}$. Bei der Bromierung mit $N$-Bromsuccinimid (NBS) in siedendem Methylenchlorid oder mit Brom in Methylenchlorid bei $0^{\circ} \mathrm{C}$ entsteht das 2-Brom-Substitutionsprodukt 2, während bei $-75^{\circ} \mathrm{C}$ Brom an 1 addiert wird. Das Dibromaddukt 3 ist nur NMR-spektroskopisch nachweisbar, wogegen das Tetrabromadduk1 4 isoliert werden kann ${ }^{5}$.

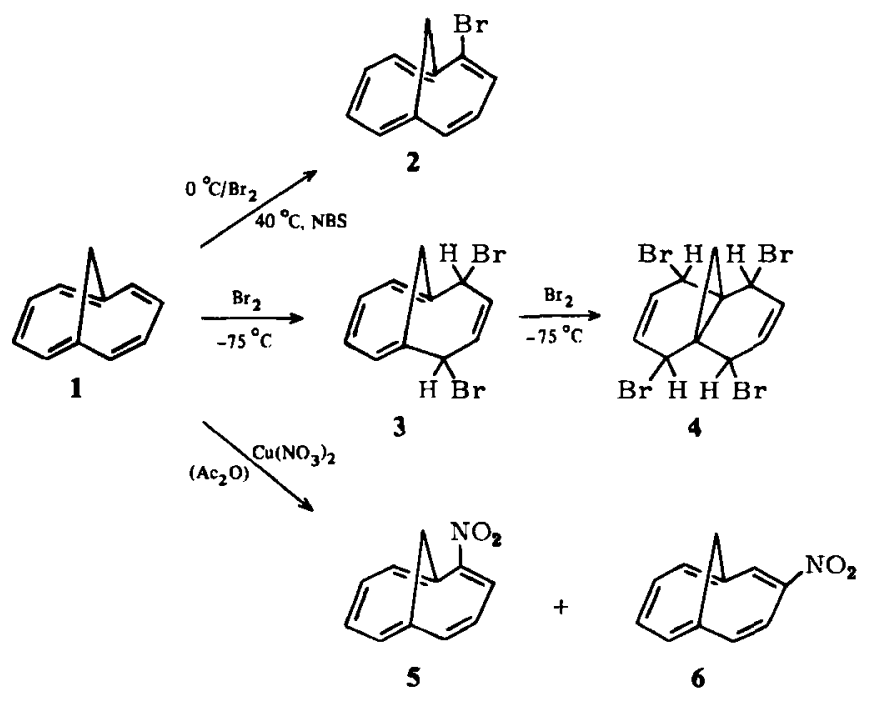

Wegen der Säurelabilität von 1 wurde seine Nitrierung mit Kupfernitrat in Acetanhydrid vorgenommen, wobei ein nur schwer trennbares Gemisch aus 2-Nitro- (5) und 3-Nitro-1,6-methano-[10]annulen (6) entsteht ${ }^{6)}$.

Um weitere Informationen über das „olefinische“ bzw. „aromatische“ Reaktionsverhalten des 1,6-Methano-[10]annulens (1) zu bekommen, untersuchten wir einerseits die Umsetzungen von 1 mit Diazonium- und Nitrosylium-Ionen als milden Elektrophilen und zum anderen die Reaktion mit Isocyanaten, die je nach Art des Substrats Cycloaddition oder elektrophile Substitution eingehen können.

Mit $p$-Nitrobenzoldiazonium-chlorid erhielten wir bei Temperaturen zwischen 0 und $40^{\circ} \mathrm{C}$ ein Gemisch, aus dem wir nach chromatographischer Trennung - allerdings nur in sehr geringen Ausbeuten - ein p-Nitrophenylazo-1,6-methano-[10]annulen und ein p-Nitrophenyl-1,6-methano-[10]annulen isolieren konnten, deren ${ }^{1} \mathrm{H}$-NMR-Spektren keine eindeutige Aussage erlaubten, ob die 2- oder 3-substituierten Verbindungen vorliegen.

Der Versuch, 1 mit Natriumnitrit in saurer Lösung bei $50^{\circ} \mathrm{C}$ umzusetzen, verlief negativ, auch mit $p$-Chlorphenylsulfonylisocyanat reagierte 1 selbst in siedendem Dichloräthan

5) E. Vogel, W. A. Böll und M. Biskup, Tetrahedron Lett. 1966, 1569.

6) E. Vogel und W. A. Böll, Angew. Chem. 76, 784 (1964); Angew. Chem., Int. Ed. Engl. 3, 642 (1964); W. Klug, Dissertation, Univ. Köln 1972. 
nicht. Dagegen erfolgte mit dem reaktiven Chlorsulfonylisocyanat bereits bei $0^{\circ} \mathrm{C}$ in Chloroform oder Methylenchlorid nahezu quantitativ Umsetzung zum 2-( $\mathrm{N}$-Chlorsulfonylcarbamoyl)-1,6-methano-[10]annulen (7). Infolge der Hydrolyseempfindlichkeit der Verbindung waren keine stimmenden Analysenwerte zu erhalten; das ${ }^{1} \mathrm{H}-\mathrm{NMR}$ Spektrum zeigt jedoch die Methylenprotonen bei $\delta=-0.61 \mathrm{ppm}$ als breites Singulett sowie die Kernprotonen bei $\delta=6.7-7.8 \mathrm{ppm}$, das IR-Spektrum die Säureamidbande bei $1710 \mathrm{~cm}^{-1}$. Außerdem ergab die alkalische Verseifung von 7 mit konz. Kalilauge in Glycol die bekannte 1,6-Methano-[10]annulen-2-carbonsäure (8).

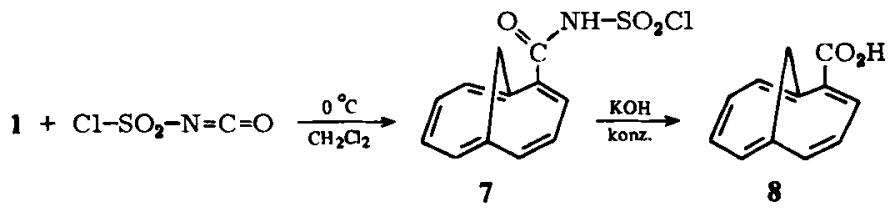

Die Reaktion, die sich sowohl NMR- wie auch IR-spektroskopisch verfolgen läßt, gibt keinen Hinweis, daß intermediär ein Cycloadditionsprodukt auftritt. Diese Substitutionsreaktion mit Chlorsulfonylisocyanat bestätigt das bevorzugte Reaktionsverhalten von 1 als Aromat.

Bei der Bromierung von 1 mit elementarem Brom bei $-75^{\circ} \mathrm{C}$ wurden die cis-Additionsprodukte 3 und 4 gefunden (s. oben). Wir versuchten nun zu klären, ob diese Verbindungen über eine synchrone Bromaddition entstehen, was aufgrund der cis-Addition durchaus plausibel wäre, oder über eine $\sigma$-Komplex-Zwischenstufe.

Der Gedanke lag nahe, die Bromierung in Gegenwart eines Konkurrenznucleophils zu untersuchen, da der Einbau eines solchen einen mehrstufigen Reaktionsablauf beweisen würde. Da sich das Dibromaddukt 3 infolge seiner thermischen Labilität in Substanz nicht isolieren läßt, ist der Beweis einer Zwischenstufenreaktion bei der Bromierung nur dann erbracht, wenn in den isolierbaren Tetrahalogenaddukten zwei Atome des Konkurrenznucleophils eingebaut werden.

Die Chlorierung von 1 mit 2 Moläquivv. Chlor bei $-70^{\circ} \mathrm{C}$ führt zu der zu 4 strukturanalogen Tetrachlorverbindung, wie wir ${ }^{1} \mathrm{H}$-NMR-spektroskopisch nachweisen konnten. Chlorid erschien deshalb als ein geeignetes Konkurrenznucleophil. Ein Halogenidaustausch in den Tetrahalogenaddukten unter den Versuchsbedingungen konnte ausgeschlossen werden.

Bei der Umsetzung von 1 mit 2.22 Moläquivv. Brom und 2.24 Moläquivv. Triäthylbenzylammoniumchlorid $([\mathrm{TEBA}]]^{\oplus} \mathrm{Cl}^{\ominus}$ ) in absol. Methylenchlorid bei $-70^{\circ} \mathrm{C}$ erhielten wir ein kristallines Gemisch von mindestens drei verschiedenen Tetrahalogenaddukten, dessen Auftrennung uns bisher nicht gelungen ist. Neben dem Tetrabromaddukt 4 lassen sich massenspektroskopisch ein Monochlortribromaddukt 9 und ein Dibromdichloraddukt 10 identifizieren. Eine Aussage über die Konfiguration der Verbindungen 9 und 10 oder deren Isomere ist nicht möglich.

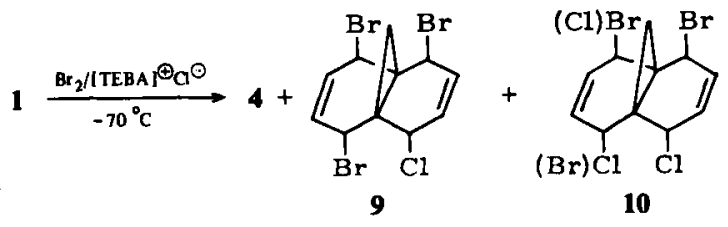


Während bei der Bildung von 9 für den ersten oder zweiten Reaktionsschritt eine synchrone Bromaddition nicht ausgeschlossen werden kann, ist die Bildung von 10 nur über zwei Zweistufenadditionen zu erklären.

Bei der Bromierung von 1 mit elementarem Brom bei $0^{\circ} \mathrm{C}$ sowie mit $N$-Bromsuccinimid bei $40^{\circ} \mathrm{C}$ wurde stets das 2-bromsubstituierte Annulen 2 erhalten (s. oben). Dies steht im Einklang mit extended Hückel- und CNDO-Rechnungen, die für die $\sigma$-Komplexstabilisierung in 1 eine deutliche Bevorzugung der 2-Position gegenüber der 3-Position zeigen $^{7}$. Im Gegensatz zur Bromierung wird für die Nitrierung ein früher Übergangszustand angenommen und die Bildung eines $\pi$-Komplexes als geschwindigkeitsbestimmend angesehen ${ }^{8 !}$.

Diese Änderung im Reaktionsmechanismus gegenüber der Bromierung kann eine Erklärung für das Auftreten der beiden isomeren 2- und 3-Nitro-1,6-methano-[10]annulene 5 und 6 (s. oben) sein. Da die Isomerenverteilung durch die Art des Anions und des Lösungsmittels entscheidend beeinflußt werden kann ${ }^{9}$, versuchten wir nun, durch Variation des Nitrierungsmittels eine Verschiebung der Isomerenverhältnisse zu erreichen. Die Nitrierungen wurden unter den in der Tab. aufgeführten Bedingungen durchgefuihrt; nach Abbruch der Umsetzungen durch Hydrolyse, Entfernen der Lösungsmittel und chromatographischer Aufarbeitung wurden die Isomerenverhältnisse gaschromatographisch bestimmt.

Nitrierung von 1,6-Methano-[10]annulen (1) zu 2-Nitro- (5) und 3-Nitro-1,6-methano-[10]annulen (6)

\begin{tabular}{|c|c|c|c|c|c|}
\hline \multirow[b]{2}{*}{ Nitrierungsagens } & \multicolumn{2}{|c|}{ Reaktions- } & \multicolumn{2}{|c|}{$\begin{array}{c}(10) \\
\text { Ausb. }\end{array}$} & \multirow{2}{*}{$\begin{array}{c}(\%) \\
\text { Gesamt- } \\
\text { ausb. }\end{array}$} \\
\hline & ${ }^{\circ} \mathrm{C} C$ & $\underset{\mathrm{h}}{\text { Zeit }}$ & 5 & 6 & \\
\hline $\mathrm{Cu}\left(\mathrm{NO}_{3}\right)_{2} / \mathrm{Ac}_{2} \mathrm{O}$ & 30 & 5 & 62 & 38 & 58 \\
\hline $\mathrm{CH}_{3} \mathrm{COONO}_{2} / \mathrm{CH}_{2} \mathrm{Cl}_{2}$ & $\begin{array}{r}-40 \\
0\end{array}$ & $\begin{array}{l}0.5 \\
1\end{array}$ & 70 & 30 & 49 \\
\hline $\mathrm{N}_{2} \mathrm{O}_{5} / \mathrm{CH}_{2} \mathrm{Cl}_{2}$ & -50 & 0.5 & 75 & 25 & 50 \\
\hline $\mathrm{N}_{2} \mathrm{O}_{5} / \mathrm{CH}_{2} \mathrm{Cl}_{2}$ & 0 & 0.5 & 80 & 20 & 55 \\
\hline $\begin{array}{l}\mathrm{NO}_{2} \mathrm{OSO}_{2} \mathrm{CF}_{3} / \text { Collidin/ } \\
\mathrm{CH}_{2} \mathrm{Cl}_{2}\end{array}$ & 40 & 3 & $>97$ & $<3$ & 60 \\
\hline $\begin{array}{l}\mathrm{NO}_{2} \mathrm{OSO}_{2} \mathrm{CF}_{3} / \text { Collidin/ } \\
\mathrm{CH}_{3} \mathrm{CN}\end{array}$ & 80 & 1 & $>97$ & $<3$ & 65 \\
\hline
\end{tabular}

Den experimentellen Ergebnissen ist zu entnehmen, daß die Nitrierungen mit Kupfernitrat/Acetanhydrid und Acetylnitrat sowie die Umsetzungen mit Distickstoffpentoxid zusammengehören. Obwohl der Reaktionsverlauf im letzteren Fall durch die Bildung von Salpetersäure im Zuge der Reaktion undurchsichtiger wird und zudem der Anteil des 2-Isomeren deutlich erhöht ist, kann man bei diesen Umsetzungen einen ähnlichen mechanistischen Reaktionsablauf annehmen, der hauptsächlich durch die Bildungsten-

\footnotetext{
7) H. Klenk, W. D. Stohrer und F. Effenberger, Chem. Ber. 109, 777 (1976), nachstehend.

${ }^{8}$ R. W. Alder, R. Baker und J.M. Brown, Mechanism in Organic Chemistry, S. $279 \mathrm{ff}$, J. Wiley and Sons, New York 1971.

9) J. G. Hoggett, R. B. Moodie, I. R. Penton und K. Schofield, Nitration and Aromatic Reactivity, Cambridge University Press 1971.
} 
denz des Nitroniumions bestimmt wird. Aussagen darüber sind aber nur schwer zu treffen, da Lösungsmittel, Anion und Temperatur verschieden sind.

Bei der Verwendung von Nitroniumsalzen sollte man aufgrund des frïheren Übergangszustandes eine begünstigte Bildung des 3-Isomeren erwarten, doch die hohe Säureempfindlichkeit von 1 macht eine Nitrierung mit diesen Elektrophilen allein unmöglich.

Cupas und Pearson ${ }^{10}$ ) berichten über hohe Substratselektivitäten bei der Nitrierung im neutralen Medium mit Nitropyridiniumsalzen. Eine ähnlich überraschend hohe Positionsselektivität stellten wir bei der Verwendung des Salzes aus Collidin und Nitronium-trifluormethansulfonat ${ }^{11)}$ fest, wobei dieses Salz den Vorteil besonders guter Löslichkeit schon in Methylenchlorid besitzt. Die praktisch ausschließliche Bildung des 2-Isomeren bei peinlich sauberem Arbeiten läßt vermuten, daß das stabile Pyridiniumsalz das Nitrierungsagens ist und dabei das Reaktionsprofil so in Richtung eines späten Übergangszustandes verändert wird, daß die $\sigma$-Komplexbildung geschwindigkeitsbestimmend wird.

Führt man die Umsetzungen mit Distickstoffpentoxid in Methylenchlorid bei $-110^{\circ} \mathrm{C}$ bis $-120^{\circ} \mathrm{C}$ durch, sind NMR-spektroskopisch neben Zersetzungsprodukten Additionsprodukte nachweisbar, die schon bei $-95^{\circ} \mathrm{C}$ Salpetersäure abspalten, unter Bildung der beiden Nitroannulene 5 und 6. Aufgrund von Kernresonanzdaten sprechen wir einem Additionsprodukt die Struktur $11 \mathrm{zu}$; das Vorliegen eines Valenzisomerengleichgewichts $11 \rightleftharpoons 12$ ist aber ebenfalls zu diskutieren.

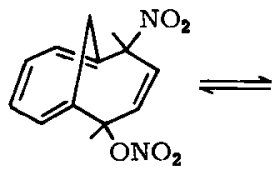

11

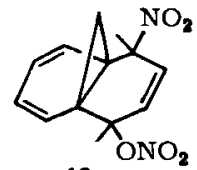

12

Bei der Umsetzung von Acetylnitrat in Methylenchlorid konnten wir keine Additionsprodukte nachweisen, da eine Reaktion erst bei höheren Temperaturen $\left(-50^{\circ} \mathrm{C}\right)$ erfolgt.

$\mathrm{Da}$ bei Nitrierungen an benzoiden Aromaten oft solche nichtkonventionellen Prozesse ablaufen, ist gut bekannt. Erst kürzlich wurde die Addition von Distickstoffpentoxid und Acetylnitrat an Naphthalin beschrieben ${ }^{121}$.

Die Bildung von 5 und 6 bei diesen Tieftemperaturreaktionen erfordert nun aber neben dem Vorliegen von 11 entweder einen ähnlichen Additionskomplex, bei dem die Nitrogruppe in 3-Position steht, oder aber teilweise $2 \rightarrow 3$-Nitrowanderung, ausgehend von 11. Solche 1,2-Nitroverschiebungen in ähnlichen Systemen sind bekannt ${ }^{12)}$. Die NMR-Spektren lassen keinen eindeutigen Beweis für ein weiteres Additionsprodukt zu, da die entsprechenden Signale durch die Absorptionen nicht identifizierter Mehrfachadditionsprodukte überlagert werden.

Der Deutschen Forschungsgemeinschaft und dem Fonds der Chemischen Industrie gilt unser Dank für die Förderung dieser Arbeit.

10) C. A. Cupas und R. L. Pearson, J. Amer. Chem. Soc. 90, 4742 (1968).

11) F. Effenberger und J. Geke, Synthesis 1975, 40.

12) Zusammenfassung: S. R. Hartshorn, Chem. Soc. Rev. 3, 167 (1974). 


\section{Experimenteller Teil}

${ }^{1}$ H-NMR-Spektren: Varian A-60 oder EM-360 der Fa. Varian Ass. (TMS als int. Standara). Massenspektren: MAT-711 der Fa. Varian Ass. Gaschromatogramme: Gaschromatograph Modell 1200 der Fa. Varian Ass. (1.7 m 5 proz. VPGS-Füllung). Säulenchromatographie: Kieselgel 60 (Fa. Merck Nr. 7734) oder Aluminiumoxid (Fa. Merck, standardisiert nach Brockmann).

p-Nitrophenylazo-1,6-methano-[10/annulen und p-Nitrophenyl-1,6-methano-/10]annulen: Zu der Aufschlämmung von $1.40 \mathrm{~g}(10 \mathrm{mmol}) p$-Nitranilin in $6 \mathrm{ml}$ halbkonz. wäßr. Salzsäure läßt man bei $34^{\circ} \mathrm{C} 0.69 \mathrm{~g}$ (10 mmol) Natriumnitrit in $4 \mathrm{ml}$ Wasser unter Rühren langsam zutropfen, versetzt anschließend mit $10 \mathrm{ml}$ Eisessig und $5.0 \mathrm{~g}$ Natriumacetat und läßt dann bei $0^{\circ} \mathrm{C} 1.42 \mathrm{~g}$ (10 mmol) 1,6-Methano-[10]annulen (1) in $4 \mathrm{ml}$ Aceton zutropfen. Nach vorsichtigem $2 \mathrm{stdg}$. Erwärmen auf $40^{\circ} \mathrm{C}$ (Wasserbad) gibt man $40 \mathrm{ml}$ Wasser zu, schüttelt mit Äther aus, trocknet die ätherische Phase über Magnesiumsulfat und engt sie nach Abfiltrieren des Trockenmittels im Rotationsverdampfer ein. Der Rückstand wird über eine Säule an $30 \mathrm{~cm}$ Kieselgel mit Cyclohexan als Laufmittel chromatographiert. Zunächst werden überschüssiges 1 und nicht identifizierte Nebenprodukte eluiert, nach $24 \mathrm{~h}$ eine gelbe Fraktion, die eingeengt und aus Methanol umkristallisiert wird. Ausb. $0.05 \mathrm{~g}(2 \%)$-Nitrophenyl-1,6-methano-[10]annulen, gelbe Kristalle mit Schmp. $124^{\prime \prime} \mathrm{C}$.

$$
\mathrm{C}_{1}, \mathrm{H}_{13} \mathrm{NO}_{2} \text { (263.3) Ber. C 77.55 H 4.98 N } 5.32 \text { Gef. C } 77.64 \text { H } 5.86 \text { N } 4.81
$$

Die in der Säule verbliebene rot gefärbte Zone wird mit Methylenchlorid eluiert und eingeengt, die schwarzen Kristalle werden aus Methanol umkristallisiert. Ausb. $0.05 \mathrm{~g}(2 \%)$-Nitrophenylazo1,6-methano-[10]annulen, Schmp. 95-98 ${ }^{\circ} \mathrm{C}$ (Zers.).

$$
\mathrm{C}_{17} \mathrm{H}_{13} \mathrm{~N}_{3} \mathrm{O}_{2} \text { (291.3) Ber. C } 70.09 \text { H } 4.50 \mathrm{~N} 14.43 \text { Gef. C } 70.09 \text { H } 4.77 \mathrm{~N} 14.90
$$

2-(N-Chlorsulfonylcarbamoyl)-1,6-methano-[10]annulen (7): Zu der Lösung von $0.71 \mathrm{~g}(5 \mathrm{mmol})$ 1 in $20 \mathrm{ml}$ absol. Methylenchlorid läßt man bei $0^{\circ} \mathrm{C} 0.71 \mathrm{~g}(5 \mathrm{mmol})$ Chlorsulfonylisocyanat in $5 \mathrm{ml}$ absol. Methylenchlorid unter Rühren zutrupfen, rührt anschließend bei Raumtemp. noch $2 \mathrm{~h}$, gibt $50 \mathrm{ml}$ absol. Cyclohexan zu, saugt die hellen Kristalle nach Abkühlen ab und trocknet sie nach Waschen mit Cyclohexan über Paraffin. Ausb. $1.3 \mathrm{~g}(92 \%)$, Schmp. $95-100^{\circ} \mathrm{C}$ (Zers.).

1,6-Methano-[10]annulen-2-carbonsäure (8): $1.4 \mathrm{~g}(5 \mathrm{mmol}) 7,20.0 \mathrm{~g}$ Kaliumhydroxid und $20 \mathrm{ml}$ Glycol werden $5 \mathrm{~h}$ zum gelinden Sieden erhitzt, nach Abkühlen mit $50 \mathrm{ml}$ Wasser versetzt und mit $20 \mathrm{ml}$ Äther ausgeschüttelt. Die wäßr. Lösung wird mit verd. wäßr. Schwefelsäure angesäuert und die ausgefallene Carbonsäure 8 zweimal mit $50 \mathrm{ml}$ Äther extrahiert. Nach Trocknen der äther. Phasen über Magnesiumsulfat und Abfiltrieren wird im Rotationsverdampfer eingeengt und der Rückstand aus Essigester umkristallisiert. Ausb. $0.73 \mathrm{~g}$ ( $78 \%$ braunrote Kristalle, Schmp. 172 bis $173^{\circ} \mathrm{C}$, Lit. ${ }^{6)} 172-173^{\circ} \mathrm{C}$.

2,5,7,10-Tetrachlortricyclo[4.4.1.0 $0^{1.6} /$ undeca-3,8-dien: $\mathrm{Zu} 0.74 \mathrm{~g}(5 \mathrm{mmol}) 1$ in $10 \mathrm{ml} \mathrm{absol}$. Methylenchlorid läßt man bei $-70^{\circ} \mathrm{C} 0.71 \mathrm{~g}(10 \mathrm{mmol})$ Chlor in $5 \mathrm{ml}$ absol. Methylenchlorid zutropfen, läßt anschließend auf Raumtemp. kommen, wäscht dann mit wenig Wasser, trocknet über Magnesiumsulfat und kristallisiert den farblosen Niederschlag, den man nach Einengen der Lösung im Rotationsverdampfer erhält, aus Methanol um. Ausb. $1.2 \mathrm{~g}(85 \%)$, Schmp. 137 bis $138^{\circ} \mathrm{C}$.

${ }^{1} \mathrm{H}$-NMR (in $\left.\mathrm{CDCl}_{3}\right): \delta=1.01\left(\mathrm{CH}_{2}-\right.$ Brückenprotonen), 5.14 und 5.5 (s für zwei $-\mathrm{C}_{\mathrm{C}}^{\prime} \mathrm{H}$ und zwei $-\mathrm{CH}=$ ), 4.8 und $5.8 \mathrm{ppm}$ (m für zwei $-\mathrm{C}_{1}^{\prime} \mathrm{H}$ und zwei $-\mathrm{CH}=$ ).

$$
\mathrm{C}_{11} \mathrm{H}_{10} \mathrm{Cl}_{4} \text { (284.0) Ber. C } 46.52 \mathrm{H} 3.55 \mathrm{Cl} 49.93 \text { Gef. C } 46.78 \text { H } 3.71 \mathrm{Cl} 49.73
$$


Bromierung von 1 in Gegenwart von Triäthylbenzylammoniumchlorid $\left([T E B A]^{\oplus} \mathrm{Cl}{ }^{\ominus}\right.$ )

a) $\mathrm{Zu}$ der Lösung von $1.42 \mathrm{~g}(10 \mathrm{mmol}) \mathrm{l}$ und $5.1 \mathrm{~g}(22.4 \mathrm{mmol})[\mathrm{TEBA}]^{\oplus} \mathrm{Cl}{ }^{\ominus}$ in $20 \mathrm{ml}$ absol. Methylenchlorid läßt man bei $-70^{\circ} \mathrm{C} 3.5 \mathrm{~g}(22 \mathrm{mmol})$ Brom in $10 \mathrm{ml}$ Methylenchlorid langsam zutropfen und läßt anschließend auf Raumtemp. kommen. Nach viermaligem Waschen der Lösung mit Wasser wird die organische Phase über Magnesiumsulfat getrocknet, nach Abfiltrieren des Trockenmittels und nach Abziehen des Lösungsmittels im Rotationsverdampfer der Rückstand aus Tetrachlorkohlenstoff umkristallisiert. Ausb. $2.2 \mathrm{~g}$ helle Kristalle, Schmelzbereich $124-128^{\circ} \mathrm{C}$.

MS (30eV, $195^{\circ}$-Quelle, $90^{\circ}$-Direkteinlaß): keine $\mathbf{M}^{+}$, aber deutlich $\left(\mathbf{M}^{+}-\mathrm{Br}\right)$. Masse (rel Int. in $\%$ :

2,5,7,10-Tetrabromtricyclo/4.4.1.0 ${ }^{1.6} /$ undeca-3,8-dien (4): 385.7 (0.38), 384.7 (1.91), 383.7 (0.55), $382.7(5.93), 381.7(0.71), 380.7(5.94), 378.7(2.1)$.

2,7.10-Tribrom-5-chlortricyclo[4.4.1.0 ${ }^{1.6}$ Jundeca-3,8-dien (9): 340.8 (1.33), 339.7 (0.8), 338.8 (7.02), $337.8(1.12), 336.8(9.65), 335.8(0.58), 334.8(4.00)$.

2-Brom-5-chlor-7(10)-brom-10/7)-chlortricyclo/4.4.1.0 ${ }^{1.6}$ Jundeca-3,8-dien (10): $294.8(0.91)$, 293.8 (0.30), $292.8(1.82), 290.8(1.05)$.

b) $\mathrm{Zu}$ der Lösung von $1.42 \mathrm{~g}(10 \mathrm{mmol}) 1 \mathrm{in} 15 \mathrm{ml}$ absol. Methylenchlorid läßt man bei $-70^{\circ} \mathrm{C}$ $3.5 \mathrm{~g}(22 \mathrm{mmol})$ Brom in $10 \mathrm{ml}$ Methylenchlorid langsam zutropfen, anschließend ebenfalls bei $-70^{\circ} \mathrm{C} 5.1 \mathrm{~g}(22.4 \mathrm{mmol})$ [TEBA] ${ }^{\oplus} \mathrm{Cl}^{\ominus}$ in $5 \mathrm{ml}$ absol. Methylenchlorid und rührt dann noch $30 \mathrm{~min}$ bei $-70^{\circ} \mathrm{C}$. Nach Aufarbeiten wie vorstehend beschrieben wurde nur 4 vom Schmp. $144^{\circ} \mathrm{C}$ erhalten, Lit. ${ }^{\text {s) }}$ Schmp. $146-147^{\circ} \mathrm{C}$ (Zers.).

Nitrierung von 1. Allgemeines zur Aufarbeitung der Gemische: Nach Reaktionsabbruch durch Hydrolyse werden die organischen Lösungen sorgfältig neutral gewaschen und die Waschwässer mit Äther oder Methylenchlorid gewaschen. Die vereinigten organischen Extrakte werden über Magnesiumsulfat getrocknet und nach Abfiltrieren des Trockenmittels im Rotationsverdampfer eingeengt. Der Rückstand wird über $30 \mathrm{~cm}$ Aluminiumoxid $(\varnothing 1.5-2.5 \mathrm{~cm})$ mit Cyclohexan/ Methylenchlorid (70/30) filtriert. Ủberschüssiges 1 wird hierbei zuerst eluiert, etwaige Polymere bleiben auf der Säule sitzen. Die gelben, nitroannulenhaltigen Lösungen werden danach eingeengt, das Isomerenverhältnis wird gaschromatographisch bestimmt.

a) Mit Kupfernitrat/Acetanhydrid: $\mathrm{Zu}$ der Aufschlämmung von $2.70 \mathrm{~g}$ (11 mmol) Kupfernitrat $\left(\mathrm{Cu}\left(\mathrm{NO}_{3}\right)_{2} \cdot 3 \mathrm{H}_{2} \mathrm{O}\right)$ in $50 \mathrm{ml}$ Acetanhydrid läßt man unter Eiskühlung $1.42 \mathrm{~g}(10 \mathrm{mmol}) 1 \mathrm{in} 20 \mathrm{ml}$ Acetanhydrid zutropfen, rührt bei Raumtemp. noch $5 \mathrm{~h}$, gibt $100 \mathrm{ml}$ konz. wäßr. Kaliumhydroxidlösung zu, kocht auf, extrahiert zweimal mit tiefsiedendem Petroläther, trocknet über Magnesiumsulfat und arbeitet auf. Ausb. $1.1 \mathrm{~g}(58 \%)$ Gemisch aus 2-Nitro- (5) (62\%) und 3-Nitro-1,6-methano$[10]$ annulen (6) (38\%).

b) Mit Acetylnitrat: $\mathrm{Zu} 1.5 \mathrm{~g}$ fein gepulvertem Silbernitrat in $20 \mathrm{ml}$ absol. Methylenchlorid gibt man bei $-50^{\circ} \mathrm{C} 0.44 \mathrm{~g}(5.6 \mathrm{mmol})$ Acetylchlorid, rührt $30 \mathrm{~min}$, dekantiert bei $-50^{\circ} \mathrm{C}$ rasch vom Silberchlorid und vom überschüssigen Silbernitrat ab und läßt bei $-40^{\circ} \mathrm{C} 0.71 \mathrm{~g}(5 \mathrm{mmol}) 1$ in $5 \mathrm{ml}$ Methylenchlorid zutropfen. Nach Stehenlassen bei Raumtemp. bis die Temp. $0^{\circ} \mathrm{C}$ erreicht hat, rührt man bei dieser Temp. noch $1 \mathrm{~h}$, hydrolysiert anschließend mit $50 \mathrm{ml}$ Wasser und arbeitet auf. Ausb. $0.46 \mathrm{~g}(49 \%)$ Gemisch aus $5(70 \%)$ und $6(30 \%)$.

c) Mit Distickstoffpentoxid bei $-50^{\circ} \mathrm{C}: 0.71 \mathrm{~g}(5 \mathrm{mmol}) 1$ in $20 \mathrm{ml}$ absol. Methylenchlorid werden bei $-50^{\circ} \mathrm{C}$ mit $0.6 \mathrm{~g}(5.55 \mathrm{mmol})$ Distickstoffpentoxid in Methylenchlorid versetzt. Nach $30 \mathrm{~min}$ Rühren bei $-50^{\circ} \mathrm{C}$ wird die rotbraune Lösung aufgearbeitet. Ausb. $0.47 \mathrm{~g} \mathrm{(50 \% )} \mathrm{Gemisch}$ aus $5(75 \%)$ und $6(25 \%)$. 
d) Mit Distickstoffpentoxid bei $0^{\circ} \mathrm{C}$ : Wie vorstehend unter c) beschrieben - jedoch bei $0^{\circ} \mathrm{C}$. Ausb. $0.51 \mathrm{~g}(55 \%)$ Gemisch aus $5(80 \%)$ und $6(20 \%)$.

e) Mit Nitronium-trifluormethansulfonat und Collidin in Methylenchlorid ${ }^{13)}: \mathrm{Zu} 0.5 \mathrm{~g}(2.56 \mathrm{mmol})$ Nitronium-trifluormethansulfonat in $10 \mathrm{ml}$ absol. Methylenchlorid läßt man bei $0.2 \mathrm{C}$ und unter Stickstoff $0.32 \mathrm{~g}(2.64 \mathrm{mmol})$ Collidin in $5 \mathrm{ml}$ Methylenchlorid zutropfen und rührt anschließend noch $30 \mathrm{~min}$ bei Raumtemp., bis vollständige Lösung erfolgt ist. Diese Lösung läßt man ebenfalls unter Stickst off und unter Feuchtigkeitsausschluß zu $0.35 \mathrm{~g}(2.5 \mathrm{mmol}) 1 \mathrm{in} 20 \mathrm{ml}$ absol. Methylenchlorid bei $0^{\circ} \mathrm{C}$ zutropfen, rührt $1 \mathrm{~h}$ bei Raumtemp., danach $2 \mathrm{~h}$ unter leichtem Rückfluß und arbeitet auf. Ausb. $0.28 \mathrm{~g}(60 \%)$ an $5(>97 \%)$ und $6(<3 \%)$.

1) Mit Nitronium-trifluormethansulfonat und Collidin in Acetonitril ${ }^{133}$ : Wie vorstehend, jedoch mit Acetonitril anstelle von Methylenchlorid als Lösungsmittel und 1 stdg. Kochen unter RückfluB. Ausb. $0.305 \mathrm{~g}(65 \%)$ an $5(>97 \%)$ und $6(<3 \%)$.

13) Geringe Spuren an Feuchtigkeit oder unsauberes Arbeiten führen zu einer Änderung des lsomerenverhältnisses $\mathbf{5 / 6}$. 